\title{
Recent Spectroscopic Evidence for Aperiodic Activity in Classical Be Stars
}

\author{
Myron A. Smith \\ Computer Sciences Corporation \& Catholic Univ. of America
}

\begin{abstract}
This review focuses on aperiodic line profile activity in several Be stars, ignoring attributes of pulsations probably occurring in any of the stars studied. Herein, I discuss spectroscopic evidence of heating (and cooling) of regions of Be star atmospheres, of line profile transients called dimples and high velocity absorptions. These features will be interpreted in terms of mass ejections (not necessarily permanent). Some of these events are violent and spasmodic, some are ordered and quasisteady, and some are associated with downflows. I close with new evidence for time-varying, corotating clouds suspended over a few Be stars.
\end{abstract}

\section{Introduction}

As the title implies, this review focuses on aperiodic line profile variability $(l p v)$. Considerable monitoring work has been done on a handful a few Be stars over the last few years. The primary goal of these studies has been to delineate the properties of their nonradial pulsations (NRPs). Even so, some of the reported $l p v$ 's appear to occur at irregular times and are not easily ascribable to pulsations. Herein I will discuss recent evidence for atmospheric heatings and cooling processes and proceed to cases where temperature changes are not indicated.

\section{Evidence of Atmospheric Heatings (and Coolings)}

It seems fitting to start by bringing to this conference's attention the Copernicus observations of $\gamma$ Cas by Slettebak \& Snow (1978). The authors demonstrated that the core of the Si IV $\lambda 1394$ (but not the $\lambda 1403$ line scanned several minutes earlier) responded to a rapid flare which was meanwhile observed in the $\mathrm{H} \alpha$ line on 1978 January 29. One of the Mg II doublet lines (but again not both) responded in a similar way an hour later. The matter is made more fascinating by the report of a strong X-ray flare in the star at the same time (Peters 1982). At about the same epoch, Marlborough, Snow, \& Slettebak (1978; "MSS") found a decrease in the Discrete Absorption Components ("DACs") of N V over an interval of three hours. To date this remarkable behavior seems neither to have been explained nor challenged. To shed light on this and related questions about X-ray emission from $\gamma$ Cas, R. Robinson and I were fortunate enough to be able to conduct a simultaneous UV, X-ray campaign using the Hubble Space Telescope GHRS and Rossi X-ray Timing Explorer to observe the star on 1996 14-15 March. 
Figure 1 depicts the orbitally averaged X-ray fluxes (rescaled and shifted) and the fluxes in absorptions lines of Si IV $\lambda 1403$, Si III $\lambda 1417.2$, and $\mathrm{Fe}$ V $\lambda 1415.1$. These lines are formed in material just beginning to move outward from the star (see Smith \& Robinson 1999; SR99). Note that the fluxes of the $\mathrm{Fe} \mathrm{V}$ line are anticorrelated with the X-ray emission. Plasma must be heated to $\geq 34,000 \mathrm{~K}$ to show this line. Since this temperature is too high for circumstellar material in radiative equilibrium near a B0.5 star, Cranmer, Smith, \& Robinson 2000 (CSR00) suggested that X-rays increase the ionization of the wind as active centers near the star's surface appear over the limb and rotate underneath it (see their Fig. 9). Consistent with this interpretation, the Si III and Si IV lines weaken with increased X-ray emission as silicon atoms ionize from $\mathrm{Si}^{+3}$ to $\mathrm{Si}^{+4}$, resulting in a weakening of the Si IV DACs. Furthermore, an examination of difference spectra from a 43-hour time series of 32 IUE observations in 1982 discloses a small-amplitude flickering of the DACs within the C IV and Si IV profiles. These excess absorptions are correlated with (unexplained) cessations of X-ray flares every $\approx 7.5$ hours (CSR00, Robinson \& Smith 2000). Each of these events can be understood if X-ray centers appear or disappear over star's limb.

This picture fits with the Copernicus results if we assume that the emissions of the lines Slettebak \& Snow observed were excited by a greater than average $\mathrm{X}$-ray flaring rate which produced caused a temporary overionizaton of the intervening wind material. However, we should not infer from any of this that X-ray illumination of disk material is typical for Be stars! Still, the example of $\gamma$ Cas shows that if X-rays are excited for any reason then absorption lines produced in nearby wind material may well show a response. One may safely assume that local regions of the star are also heated by such activity.

The idea that heatings on a classical Be star can be revealed by line emissions from disk material has been gaining popularity. Oudmaijer \& Drew (1997) discussed a rapid event in HD 76534 which raised this possibility, but new information (see below) suggests that atmospheric cooling is more likely. On perhaps surer footing are correlated emissions noted by Smith et al. (1997a) for $\lambda$ Eridani (B2e) over one day in the lines of He II $\lambda 1640, \mathrm{He} \mathrm{I} \lambda 6678$ and $\mathrm{H} \alpha$. The He II line is certainly formed only very close to the star's surface. Emission in this line is easily explained by atmospheric heating (Auer \& Mihalas 1972, Smith et al. 1997b). Model atmospheres show that the Lyman continuum flux emitted by an early B star is very sensitive to changes in the surface temperature. Thus, $\mathrm{H} \alpha$ emission strength becomes a measure not only of the disk's mass but of the star's instantaneous $\mathrm{T}_{\text {eff }}$. This argument is persuasive because it is essentially a restatement of the tried and tested Zanstra mechanism in which each Lyman continuum photon absorbed by a nebula is reemitted as a Balmer line.

The most common type of line profile transient in $\lambda$ Eri is the "dimple," defined as a quasi-absorption feature flanked by weak emissions in a He I line profile (Smith \& Polidan 1993, "SP93"). Dimples appear at irregular intervals, developing in only $\sim 20$ minutes, and lasting $\sim 2$ hours. By taking the development timescape and comparing the perturbed and total line strengths of the line, one finds that whatever causes them propagates across a region of the disk at a rate comparable to the escape velocity at the surface. Sometimes two or even three may develop at the same time and, so far, at unpredictable times. 


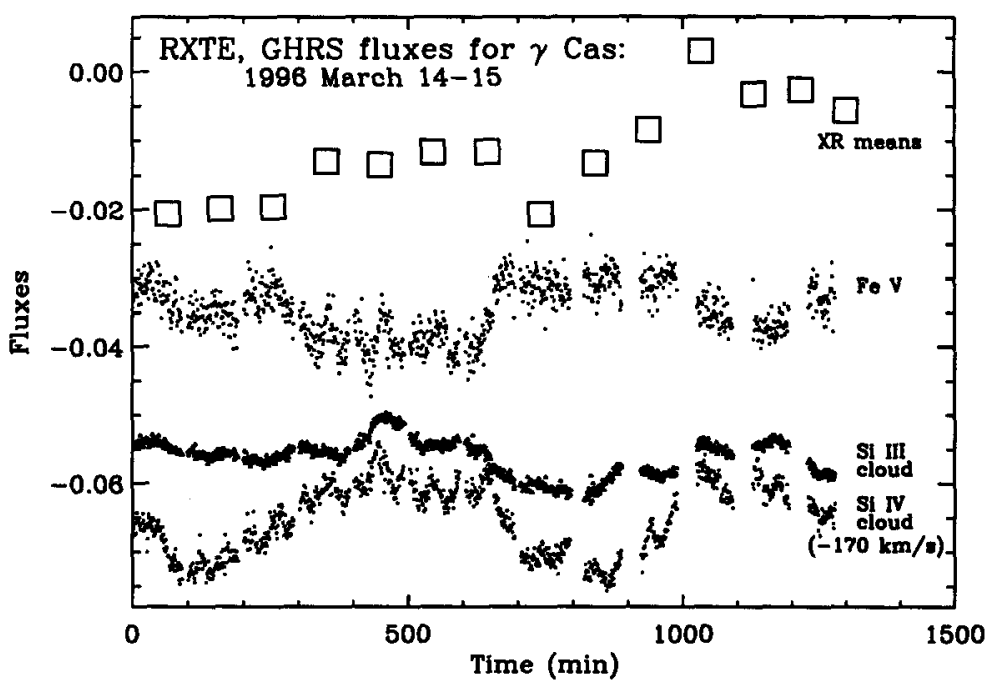

Figure 1. Comparison of X-ray light curve (orbit-bins) with simultaneous fluxes in Fe V $\lambda 1413$, Si III $\lambda 1417$, and Si IV $\lambda 1403$ lines

SP93 found dimples in lines of at least four other mild Be stars. Other authors have reported them in $\mu$ Cen's $\lambda 6678$ line as well. Optical/UV observations of these stars suggest that dimples are accompanied by a weakening of the C IV resonance lines (Smith et al. 1996; "S96)). Since C IV is formed in both the photospheres and winds of early-type Be stars, this result alone does not have a unique interpretation.

However, further observations of $\lambda$ Eri demonstrate that dimples in its $\lambda 6678$ line correlate with line weakenings of $\lambda 1640$. These weakenings are sometimes so rapid that they have to be caused by emission - the core of the intrinsic line profile must then be raised above the continuum. From the helium line modeling results cited above, the photospheric regions in which these weakenings are formed are undoubtedly heated. A peculiar " $\lambda 6678$ flare" reported by $S 97 \mathrm{~b}$ (see their Fig. 3) in $\lambda$ Eri on 1995 Sept. 12 may be related to this phenomenon. This event was first observed as a square-topped emission $13 \%$ above the continuum. Within 10 minutes it had decayed to $1 / e$ of its maximum, and in another 10 minutes it was gone. Assuming that the line emission and decay was caused by recombination, one can use the decay timescale to determine a plasma density of $N_{e} \approx 10^{11} \mathrm{~cm}^{3}$. Because this timescale and density are consistent with the "slab" structures which are believed to cause dimples (SP93), I suggest here that this event occurred near the limb and manifested the ejection of a dimpleproducing slab of matter at the limb of the star as transient line emission. If so, since the material was already in the process of cooling from recombination, the ejected gas must have initially been hotter than the photosphere.

While these various lines of evidence suggest that dimples are associated with local atmospheric heatings, some confirmations are needed. One check 
might be to search for dimple signatures in cool-ion lines such as $\lambda \lambda 6347-71$. The heating hypothesis predicts that dimples should not appear in Si II lines.

In these proceedings $\mathrm{D}$. Cohen discusses the implications of $\mathrm{X}$-rays from B stars. $\lambda$ Eri provides an interesting case because of a seven-fold increase in X-ray flux observed by Rosat (Smith et al. 1993). The appearance of this "flare" again suggests that something can occasionally heat this star's surface. A few astronomers have asked me privately whether this event might have been caused by a strong wind-wind shock interactions or by a flare from a cool active companion. Yet $\lambda$ Eri was in a normal X-ray but weak wind state just prior to and after the flare. This fact argues against the flare accompanying changes in the wind conditions. The problem with the cool-companion flaring idea is the absence of signatures of three strong Fraunhofer lines in $\lambda$ Eri's spectrum (Smith et al. 1997b), thereby making it unlikely that a cool companion can exist even six magnitudes fainter than the Be star. Thus, the flaring hypothesis seems so far to have survived scrutiny. Additionally, weak fluctuations in the X-ray output make it possible that low-level events are common in this star (Smith et al. 1997b).

Cohen, Cassinelli, \& MacFarlane (1997) found that $\mu$ Cen, another highly active star in the optical and UV regions, exhibited "harder" X-ray emission $\mathrm{X}$-ray spectra observed in other B stars, plus it may well be variable. Although the evidence for X-ray variability and therefore surface activity is limited to two Be stars, this limited record could be due in part to the comparatively little observational effort devoted to Be stars with X-ray instruments.

How about evidence for atmospheric coolings? This too has been well documented, but sometimes the interpretation is ambiguous. Still, the arguments in each case have been different, which makes it easier perhaps to accept - how can an atmosphere be cooled?! Let us hope that the razor overhanging these claims is owned by Occam and not Damocles.

Consider one such case, $88 \mathrm{Her}$. This is a Be star known for its transitions among normal/Be/Be-shell phases. Barylak \& Doazan (1986) and Doazan et al. (1986) discovered panchromatic $(\lambda \lambda 1500-5500)$ flux decreases in this star amounting to $31 \%$ from 1976 to 1979 (probably mostly in one year). At the same time the star made a transition from a B- "normal" to a Be-shell stage. During the next few years of the shell ejection phase, the fluxes gradually increased to their pre-outburst levels. Because both the Balmer and Paschen continua were affected, the authors argued that the flux decreases could not be caused by an opacity-related redistribution of flux. Rather, the changes were necessarily caused by a year-long decrease in the star's luminosity, which because of accompanying color changes must be due to a decrease in the star's $\mathrm{T}_{\text {eff }}$ of at least $1000 \mathrm{~K}$. Put another way, it looks like the star's subphotosphere somehow could have dammed up and subsequently reradiated its pent-up flux. Could this energy be converted to the mechanical energy needed to lift off the shell that formed at that time?

Moving to shorter timescales, Rivinius et al. (1998; "R98") documented a drop in $\mathrm{H} \alpha$ emission strength from $\mu$ Cen (B2e) in the few days prior to a new outburst. They suggested that the star's Lyman continuum flux emitted had decreased and reduced the photoionization of the disk, resulting in less $\mathrm{H} \alpha$ emission. This "Zanstra argument" was first used compellingly by Oudmaijer \& 
Drew (1997) who suggested that changes in the Lyman continuum flux of HD 76534 (B2e) caused a rapid decrease and increases in this star's $\mathrm{H} \alpha$ emission strength on 1995 January 9-11. However, further study of the HIPPARCOS and spectroscopic archives for HD 76534 has disclosed that the star's "normal" $\mathrm{H} \alpha$ emission has been at the higher level all along (Oudmaijer \& Drew 1999). This readjustment in our understanding of this event demonstrates that something happened to decrease the photoionization of the disk from January 9 to January 11 and then to increase it again within 2.5 hours on January 11. Thus, the star's (sub)photosphere may have blocked the emergence of flux for a day or two, resulting in a cooling of the star's surface and a reduction in the emission of hydrogen-ionizing photons.

For completeness, one should also add the case of $\rho$ Leo (B1 Iab), even though it is not a Be star. Smith \& Ebbets (1981; SE81) reported a 60\% decrease in the strengths of the Si III triplet lines in 2.5 hours on 1979 February 16. This change was accompanied by a strengthening of the line's wings. To explain this, SE81 suggested that the atmospheric continuous opacity had shifted to electron scattering. Such a change could be explained by an atmospheric cooling of $\geq 1,000 \mathrm{~K}$. SE81 also noted that increases in $\mathrm{H} \alpha$ emission, which arise from low-velocity mass ejections, are correlated with decreases in the Si III strengths. Indeed, within hours of the Si III event on February 16th the $\mathrm{H} \alpha$ line exhibited emission after showing none on the previous night.

The case of $88 \mathrm{Her}$ seems the strongest of these examples: it is difficult to avoid the conclusion that the atmosphere's temperature has decreased when coherent panchromatic flux changes occur. Including the other cases as well, the evidence supports the notion that a subphotospheric energy reservoir can make itself felt over a variety of time scales in a Be star's outer envelope. One must consider the possibility that transient dammings of flux cause releases of energy which trigger ejections. We note the additional surprise that these coolings and ejections do not seem to be confined to small regions on these stars' surfaces.

What could cause flux blocking? Solar output fluctuations bring to mind the Sun as an example. It is well known that magnetic flux complexes cause solar output fluctuations on on 27-day and 11-year cycles (Lean 1997) as they first appear and evolve on the Sun's surface Also, solar-type stars without activity cycles are fainter that their active counterparts, suggesting that magnetic-output correlations exist over longer timescales (Baliunas \& Jastrow 1990). It is not known what mechanisms are responsible for these relations, but flux transport through or around magnetic bubbles is probably somehow involved. Yet one should not get too carried away with such comparisons: (1) I am making unsubstantiated inferences here that subsurface magnetic complexes exist in Be stars, and (2) we does not know that flux transport is altered in the same ways by magnetic structures in convective and nonconvective environments.

\section{High Velocity Absorption Features \& Coordinated Flows}

hva's: The term "high velocity absorptions" (hva) has been used for B supergiants to indicate transient absorptions which appear near either edge of the star's Balmer line profiles (Kaufer, these proc.). R98 recently applied this term to He I features in $\mu$ Cen. These authors observed an hva at the start of an 
outburst in 1995 this star and also on 2 nights the next season. These are shown in Figure 2. R98 conjectured that hva's accompany mass loss transfers to the star's disk, and they also suggested that the event which appeared in only 12 minutes in the $\lambda 6678$ line on an earlier occasion (Peters 1986) was also an hva. In her discussion of unusual activity of during another outburst, Peters (1998) highlighted yet another such feature.

Motivated by these examples, I searched through my own archives of $\lambda 6678$ line profiles of $\lambda$ Eri and indeed found several examples of $h v a$ 's. These examples suggest average durations ranging from a few to many hours (one day?) The examples of hva's which Peters and I have found that they appear on a severalminute time scale of dimples but probably last much longer than dimples do. In addition, so far I have found that hva's are always accompanied by emissions at another velocity, generally redshifted by a few hundred $\mathrm{km} \mathrm{s}^{-1}$. In the few cases when hva's have been observed to develop, the emissions appeared at the same time. Unquestionably, hva's are signatures of mass outflow. The issue is whether the redshifted emission arises from a downflow of material impacting the surface or an outflow from the star also directed away from the observer.
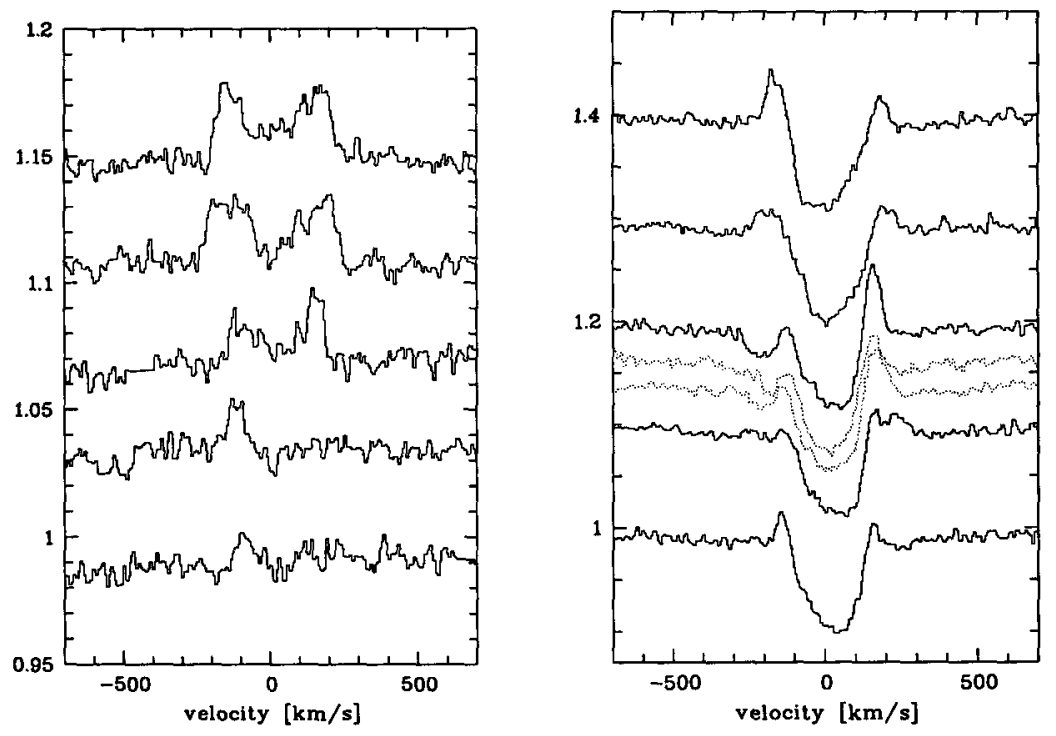

Figure 2. HEROS sequence of Si II (left) and He I (right) lines in $\mu$ Cen, showing an hva in middle He I spectra. Dotted lines show hva's on nights a few months later (from Fig. 5 of Rivinius et al. 1998; courtesy, T. Rivinius et al.)

To address this question I made use of HEROS (echelle) data kindly provided by $\mathrm{T}$. Rivinius for the night immediately preceding as well as of the night of an hva event itself (Figure 2). I modeled the absorptions and emissions in 
their second and third spectra (from bottom of Figure 2, shown above) in the Si II $\lambda 6347$ and He I $\lambda 6678$ lines with the Hubeny CIRCUS programs (Hubeny \& Heap 1996). This program first computes the synthesized spectrum for a stellar atmosphere and determines the flux added or subtracted by a homogeneous cloud of specified parameters either occulting the disk or viewed over the limb of the star. For these observations the presence of emission in the Si II ions suggested that the He I emission is not due to non-LTE effects in the He I atom, so I placed the putative cloud in CIRCUS off the limb of the model star. Next, I determined model parameters leading to a fit of both $\mathrm{He} \mathrm{I}$ and $\mathrm{Si}$ II emissions in the spectrum which also showed the hva component. The opposing sensitivities of Si II and He I permit a good determination of the temperature of the circumstellar matter in the line forming region. Thus, for reasonable densities (volume: $10^{12} \mathrm{~cm}^{-3}$; column: $10^{23} \mathrm{~cm}^{-2}$ ), the redshifted emissions of the two ions are probably produced by material having a temperature $\mathrm{T} \approx 11,500$ $\pm 200 \mathrm{~K}$. This low temperature rules out the "downflow alternative" but suggests rather that the hva is absorbed by an outflowing structure, spewing material at a moderate velocity. Angular momentum conservation requires that this outflow take on a spiral pattern, cooling and probably accelerating as it leaves the star (alternatively, it could represent an ionization front). One cannot tell whether this flow augments the disk mass, but our exercise does support R98's conjecture that hva's accompany the outburst process.

Another result from this modeling is that the hva feature itself is formed in a narrow temperature range of $16,000 \pm 1000 \mathrm{~K}$ at the star's surface. This implies that the event itself is not a part of a virializing process which generates heat and mechanical energy. Therefore, it precludes certain heat-generating mechanisms (e.g. shocks) which might otherwise be invoked to drive this mass flow.

Coordinated flows: Kaufer has reviewed evidence for outflows and inflows on B supergiants from their hva's. In $\beta$ Ori (B8 Iab) either or both blue- and red-edge $h v a$ 's can be observed at the same time. Israelian et al. (1997) observed an infall feature nearly half a rotation period after an upflow, leading them to postulate a collimated flow attributable to magnetic loops. The concept of returning infall as the outcome of ballistically controlled "failed ejections" was raised in the case of $\lambda$ Eri by Smith, Peters, \& Grady (1991; SPG91) who observed the evolution of a red-edge hva over a few hours. This author has also reported (Smith 1989, see Figs. 36 \& 37) "flows" of emission moving continuously from the blue to red edge of this line over several hours. The flows have the appearance of being coherent and are therefore consistent with the magnetic loop conjecture.

The problem with mass-tossing (non-magnetic) scenarios is that ballistic material must return with a range of velocities and over a variety of times and places on the star, making it more difficult to observe compared to the initial ejection. In contrast, the red-edge $\lambda 6678$ emission component was observed in $\lambda$ Eri more frequently than the blue component during the late 1980's and early 1990 's. Thus it looks unlikely that these events are products of HD instabilities or explosions. Peters (1998) came to a similar conclusion based on her study of unusual activity in this line for $\mu$ Cen on the night of 1994 April 5. This activity took place in three discrete wavelength places on the profile (see Fig. 3), with emission in one place apparently moving to another. There may be a clue here 


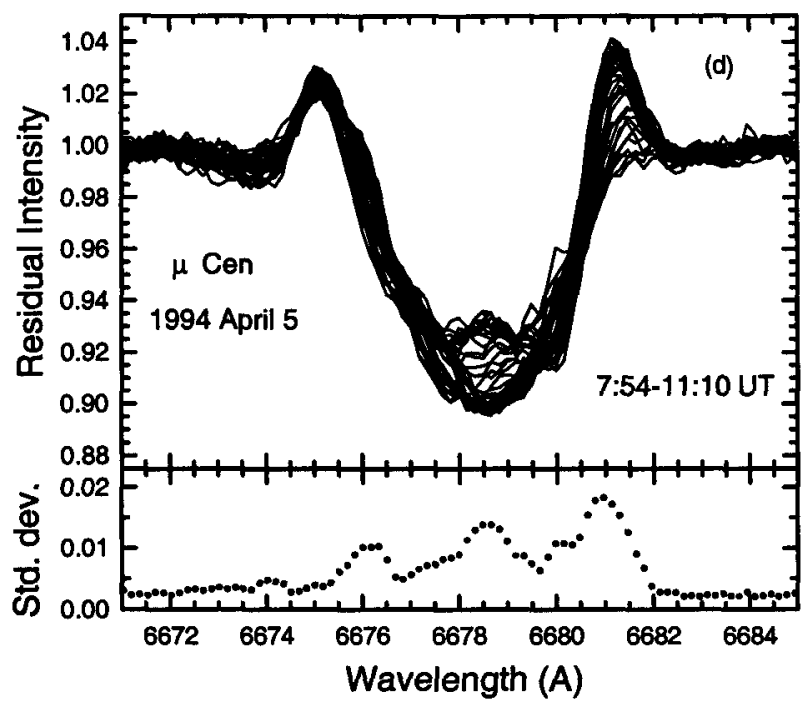

Figure 3. He I $\lambda 6678$ line activity in $\mu$ Cen on an extraordinary night: note specific wavelength regions of activity (courtesy, G. Peters)

as to the nature of the ejections of material which replenish disks in these two Be stars' outbursts.

Lacking in the record is an understanding of whether these flows are associated with heatings like dimples, thermal-neutral events like hva's, or coolings as in the case of $88 \mathrm{Her}$. In the case of $\lambda$ Eri, the greater frequency of emissions in the red over the blue edge of the He I line implies that ejected material returns to the star at high velocity and causes shocks. Separate evidence exists that surface shocks occur on some occasions (SPG91). The question of heating associated with R-emission events can be settled by repeated monitoring of such activity with an echelle system and observing the responses of low-excitation lines to transient V-component events in He I lines.

\section{Co-rotating Clouds on Be stars}

A number of He-rich magnetic Bp stars possess corotating clouds caused by trapping of wind material within magnetic surface structures. These stars, e.g. $\sigma$ Ori E and HD 37776 (Short \& Bolton 1994, Shore \& Brown 1990), show C IV resonance line strength modulations consistent with the magnetic/rotation periods. This phenomenon appears to extend to the O7V star $\theta^{1}$ Ori $\mathrm{C}$ and is observable as variable $\mathrm{X}$-ray emission (Gagné et al. 1997). Recently Smith, Robinson, \& Hatzes (1998; SRH98) have found that the short dips in the UV continuum flux curves of $\gamma$ Cas can be best attributed to cool clouds. Additional 

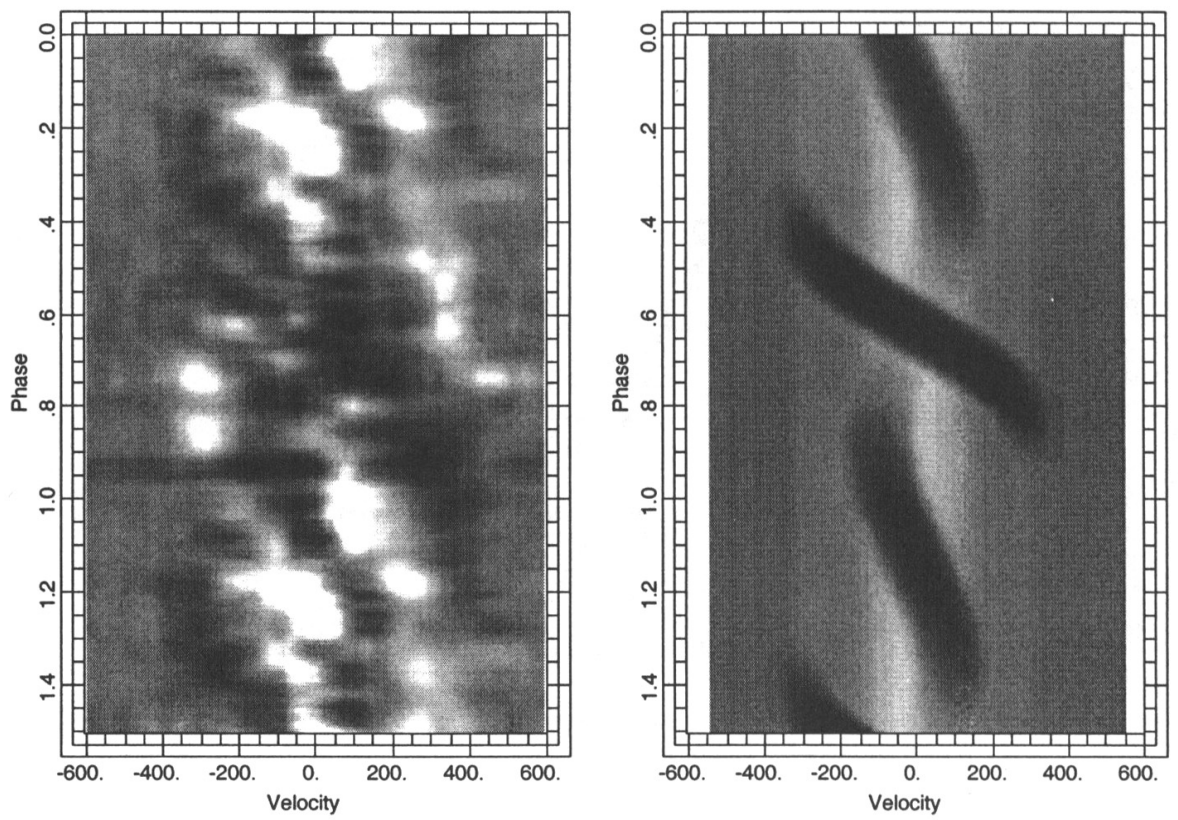

Figure 4. L. Balona's grayscale depictions of (panel a):) difference profiles through an assumed rotation cycle of $\mathrm{P}=0.78$ days and (panel b): simulated effects of two black clouds over star's surface

spectroscopic evidence appears as increased absorption in lines of cool- and hottemperature ions at times of cloud occultations (SR99).

Balona (1999a, 1999b) has just enlivened this field by suggesting that previous interpretations that NRP is the cause of photo-/spectroscopic variability in $\eta$ Cen, $\zeta$ Tau, and maybe other Be stars are in error. In his picture, both stars require two clouds centered over points on the star's surface with different longitudes and latitudes. Balona discusses the evidence for clouds in the case of $\eta$ Cen in his presentation, so I will restrict myself to $\zeta$ Tau without endorsing or challenging his other cases.

Figure 4 (left panel) shows a grayscale depiction of difference spectra from several He I lines of a nice timeseries of $\zeta$ Tau obtained by Kaye \& Gies (1997). The right-hand panel shows a crude model depicting the results of placing two spots (read: clouds) at different latitudes and longitudes on a model star disk and computing the effects on their rotational modulation on a line profile. The extended, gently sloped feature in the middle can reproduced by the transit of a cloud at the equator, while the small-amplitude sinusoidal feature originates from another at high latitude. Confirmation of such patterns is needed, but so far the resemblance of Balona's model to these observations of $\zeta$ Tau is striking.

Perhaps one confirmation of Balona's picture for $\zeta$ Tau is already available. As part of an undertaking to examine the cloud hypothesis in B stars, I have used the CIRCUS program to compute UV spectra absorbed from stellar flux for 
cloud models having various temperatures and other parameters (Smith, these proc.). I compared these computed spectra to ratios of "high flux" to "low flux" echelle spectra of Be stars which have been observed in various observing campaigns with the IUE. I find that the prototypical Bp star $\sigma$ Ori $\mathrm{E}$ gives excellent agreement with reasonable cloud parameters, thereby demonstrating a proof of concept of the technique. The match with $\zeta$ Tau data is not quite as good, but it still shows the same unmistakable signatures (monotonic rise of absorption toward short wavelengths, with a sharp rise in the $\lambda 1200$ region) of a cool absorbing medium. I conclude that this spectrum is consistent with the passage of a cool $(T=13,000 \mathrm{~K})$ cloud in front of the star and is inconsistent with a medium whose temperature is determined by radiative equilibrium.

I have raised the subject of clouds in this discussion of aperiodic activity because these clouds may not always show the same continuum absorption strength. UV light curves generated from IUE spectra of $\gamma$ Cas (Smith \& Robinson, this proc.) strongly suggest that cloud properties can change greatly from cycle to cycle. Similarly, cycle-to-cycle X-ray light curves of this star do not track very well either. If these ideas are correct, the reproducibility of light and line profile modulations from cycle to cycle may become a new model determinant between clouds and NRP for single period Be stars.

\section{Conclusions}

The atmospheric instability(ies) leading to episodic ejections of mass in Be stars goes to the seat of what constitutes the Be phenomenon. Studies of aperiodic events to date show promise in finding our what lies behind this instability. This is true whether the events are truly aperiodic events or whether there are regularities behind them, e.g., due to beating, that we have not yet discovered. Safe conclusions so far are that: (1) aperiodic spectroscopic phenomena are common to Be stars both with and without NRPs ( $\gamma$ Cas is certainly one in the latter category) and, (2) unsurprisingly, unknown temperatures and geometries lead to completely ambiguous kinematic interpretations. From Mathys's paper, it is clear that reports of direct magnetic field detections are not ever likely to be routine for Be stars. Thus, to make progress we will need to pursue the study of magnetic proxies.

One promising strategy is to monitor temperature-sensitive lines (and for some purposes, continua). An ideal way of obtaining such diagnostics would be to launch another IUE, but that is not likely in the foreseeable future. The best tools at hand are the newly commissioned optical echelle spectrographs which can monitor a wide range of wavelengths at the same time. We will lack then only a strong He II line in the optical region.

In this paper we have reviewed several types of aperiodic line profile activity in classical Be stars. Some profile variations are accompanied by changes in thermal conditions while others are not. The births of events which are temperature-neutral are particularly interesting because they imply that the mechanism causing them has found a way of channeling energy to bulk motions without dissipative heating. Such processes could be related to "evaporation" of solar chromospheric particles to the tops of prominences. Photospheric shocks, e.g., accompanying pulsations, would be expected to generate mild heating and 
thus enhanced far-UV (but not X-ray) emission. Observations of returning matter would be of great interest because they could disclose whether ejecta follow ballistic trajectories. Such events also provide thermal constraints and hence clues to how the returning matter impacts the star's surface. Let's agree that these matters are important enough to write strong observing proposals and to keep telescopes in business in order to implement them.

Acknowledgments. I thank Drs. Luis Balona, Gerrie Peters, and Thomas Rivinius for providing three of the figures displayed in this paper.

\section{References}

Auer, L. H., \& Mihalas, D. M. 1972, ApJS, 24, 193

Balona, L. A. 1999a, MNRAS, 306, 407

Balona, L. A. \& Kaye, A. B. 1999b, ApJ, 521, 407

Baliunas, S., \& Jastrow, R. 1990, Nature, 348, 520

Barylak, M., \& Doazan, V. 1986, A \& A, 159, 65

Cohen, D. H., Cassinelli, J. P., \& MacFarlane, J. J. 1997, ApJ, 487, 867

Cranmer, S. R., Smith, M. A., \& Robinson, R. D. 2000, ApJ, 537, 433

Doazan, V., Thomas, R. N., \& Barylak, M. 1986, A \& A, 159, 75

Gagné, M. Caillault, J.-P., Stauffer, J. R., \& Linsky, J. L. 1997, ApJ, 478, L87

Hubeny, I. \& Heap, S. R. 1996, ApJ, 470, 1144

Israelian, G., Chentsov, E., \& Musaev, F. 1997, MNRAS, 290, 521

Kaye, A. B., \& Gies, D. R. 1997, ApJ, 482, 1028

Lean, J. 1997, Ann. Rev. Atron. Ap., 33, 33

Marlborough, J. M., Snow, T. P.. \& Slettebak, A. 1978, ApJ, 224, 157 (MSS).

Oudmaijer, R., \& Drew, J. 1997, A \& A, 318, 198

Oudmaijer, R., \& Drew, J. 1999, A \& A, 350, 485

Peters, G., J. 1982, PASP, 94, 157

Peters, G., J. 1986, ApJ, 301, L61

Peters, G., J. 1998, PASP, 502, L59

Rivinius, Th., Baade, D., et al. 1998, A \& A, 333, 125

Shore, S. N. \& Brown, D. N. 1990, ApJ, 365, 665

Short, C. I., \& Bolton, C. T. 1994, Pulsation, Rotation, \& Mass Loss in EarlyType Stars, ed. B. Balona (Dordrecht: Kluwer), p. 171

Slettebak, A. \& Snow, T. P. 1978, ApJ, 224, L127 (SS78)

Smith, M. A., \& Ebbets, D., 1981, ApJ, 247, 158

Smith, M., Grady, C., \& Peters, G. 1991, ApJ, 367, 302

Smith, M., Grady, C. , Peters, G. , \& Feigelson, E. 1993, ApJ, 409, L49

Smith, M. A., Cohen, D. et al., 1997a, ApJ, 481, 467

Smith, M. A., Murakami, T. et al., 1997b, ApJ, 481, 479

Smith, M. A., Plett, K. et al. 1996, ApJ, 481, 479 (S96)

Smith, M. A. \& Polidan, R. S. 1993, ApJ, 403, 323 
Smith, M. A., \& Robinson, R. D. 1999, ApJ, 517, 866 (SR99)

Smith, M. A., \& Robinson, R. D. 2000, ApJ, in press

Smith, M. A., Robinson, R. D., \& Hatzes, A. P. 1999, ApJ, 507, 945

\section{Discussion}

L. Balona: It should be noted that what appear to be isolated active events in line profiles taken at arbitrary times could possibly be due to activity in clouds which corotate with the star.

M. Smith: In principle, I agree. If one takes a look at the kinds of line profile transients, one discovers this might be true for some of them (e.g. the hva's) but not for others (e.g. dimples). Dimples appear at seemingly random times, even 2-3 along the profile at the same time. From my modeling of the Rivinius et al. hva event, it appears that an hva arises from something akin to a geyser rooted on the surface of $\mu$ Cen, but I suspect this outwelling might turn on and off at irregular intervals. In that case, hva's could have both a periodic and stochastic component. As for clouds per se, I suspect that they are always present on those stars which have them and that the cloud densities, volumes, and/or temperatures change. Such changes can cause changes in their absorption amplitudes. Even so, I wouldn't characterize them as "line profile transients" (but they are probably related to "submigrating features").

J. Kubat: Can you be more specific concerning the parameters in your cloud models?

M. Smith: Initial details of our cloud models for $\gamma$ Cas were given in the Smith, Robinson, \& Hatzes paper, with modifications in the Smith \& Robinson (1999) paper. Basically, we assumed spherical clouds and attempted to constrain the flux attenuations, cloud radii, cloud heights, and permissible latitude ranges on the star's surface where they are rooted. We found heights and radii of about $0.2-0.3 \mathrm{R}_{*}$ and a transmission at $\lambda 1400$ of $\approx 80 \%$. The temperatures in 1996 were $7000-8000 \mathrm{~K}$. It appears that in 1982 the clouds were much warmer than this (Smith, these proc.). We also know that in March, 1996 the clouds had a patchy density distribution: some lines of sight have optical depths of $10^{4}$ in the Si IV line and others having depths of $\sim 1$. The higher depths probably correspond to hydrogen column densities of $\sim 10^{22} \mathrm{~cm}^{-2}$ and therefore to number densities of $10^{11} \mathrm{~cm}^{-3}$ or higher. These clouds are potentially observable as variable $\mathrm{H} \alpha$ emission. 